\title{
INFERING AN ONTOLOGY OF SINGLE CELL MOTIONS FROM HIGH-THROUGHPUT MICROSCOPY DATA
}

\author{
Alice Schoenauer Sebag ${ }^{\star \dagger} \quad$ Sandra Plancade Céline Raulet-Tomkiewicz $^{\dagger}$ \\ Robert Barouki ${ }^{\dagger} \quad$ Jean-Philippe Vert ${ }^{\star} \quad$ Thomas Walter $^{\star}$ \\ ${ }^{\star}$ MINES ParisTech, PSL-Research University, CBIO-Centre for Computational Biology, \\ Institut Curie (Paris), and U900 (INSERM, Paris), France \\ $\dagger$ UPD Univ Paris 05 (Paris) and UMR-S 1124 (INSERM, Paris) \\ ${ }^{b}$ Mathématiques et Informatique Appliquées (INRA, Jouy-en-Josas) \\ \# Agro ParisTech (Paris)
}

\begin{abstract}
Cellular motility is a fundamental biological process. Progress in the fields of gene silencing and high-throughput (HT) microscopy provide us with the tools to study its molecular basis and potential perturbators.

The primary contribution of this paper is to present MotIW, a generic workflow for single cell motility study in HT time-lapse screening data. We successfully apply it to a simulated screen, as well as a genome-wide screen. Furthermore, MotIW enables the identification of eigth motility patterns into which all trajectories from this dataset divide up into, without any prior model of cell motion.
\end{abstract}

Index Terms - High-content (high-throughput) screening, pattern recognition and classification, data mining

\section{INTRODUCTION}

Cell motility plays a key role in many physiological processes including embryonic development or immune response, and is also involved in tumor invasion and metastasis. It is a complex cellular process, regulated by many genes in multiple signaling pathways.

Many assays have been specifically designed to study cell migration mechanisms They typically consist in a perturbation (e.g. gene overexpression or downregulation) and a measure of the resulting phenotype in terms of cellular motility. Examples include analysis of cell traces on coated layers while overexpressing genes of interest [1], wound healing assays for the investigation of gene silencing effect on cell population migratory behaviour [2], or cell tracking in low-throughput time-lapse experiments while silencing key genes [3]. However, a systematic study of single cell motility has not yet been performed at a genome-wide scale.

Thomas Walter has received funding from the European Community's Seventh Framework Programme (FP7/2007-2013) under grant agreements number 258068 (Systems Microscopy). This work was partially funded by grant SMAC-ERC-280032 of the European Research Council.
The contribution of this paper is to present MotIW (Motility study Integrated Workflow), and its application to motility gene discovery. A generic methodological framework, MotIW enables to quantitatively study cell motility at single cell resolution in HT data. It consists of cell tracking, cell trajectory mapping to an original feature space, and outlier experiment detection (cf figure 1). We show the power of our method by applying MotIW to simulated data, which allows us to estimate recall and precision to be expected on real data. We then apply this workflow to a previously published genome-wide RNAi ${ }^{1}$ screen, the Mitocheck dataset. It is composed of 200,000 videos of human cell populations, each of them showing the outcome of a single gene knockdown [4]. We thus identify a list of genes whose silencing impacts cell motility and which therefore might play a role in this process. On top of that, MotIW also produces an ontology of cell trajectories in this dataset: without any prior assumption on cell motion, we are able to identify eight types of cell trajectories. The remainder of this paper is organized as follows : Section 2 gives a brief presentation of MotIW, and Section 3 discusses its application to simulated data and the Mitocheck screen.

\section{WORKFLOW OVERVIEW}

In this section, we present MotIW, our workflow for the automatic and quantitative analysis of video sets from timelapse microscopy-based screens. Figure 1 summarizes its different steps. Briefly, for each video, object segmentation and features are extracted using methods we have previously published and which are implemented in Cell Cognition ${ }^{2}$ [5]. Cells are tracked using a new machine-learning based tracking procedure, described in section 2.1. The trajectories

\footnotetext{
${ }^{1}$ RNA interference: a technique which uses small interfering RNA (siRNA) for gene silencing.

${ }^{2}$ An open-source software, developed in collaboration with the IMBA (Vienna, Austria). It is publicly available at http://cellcognition.org/.
} 


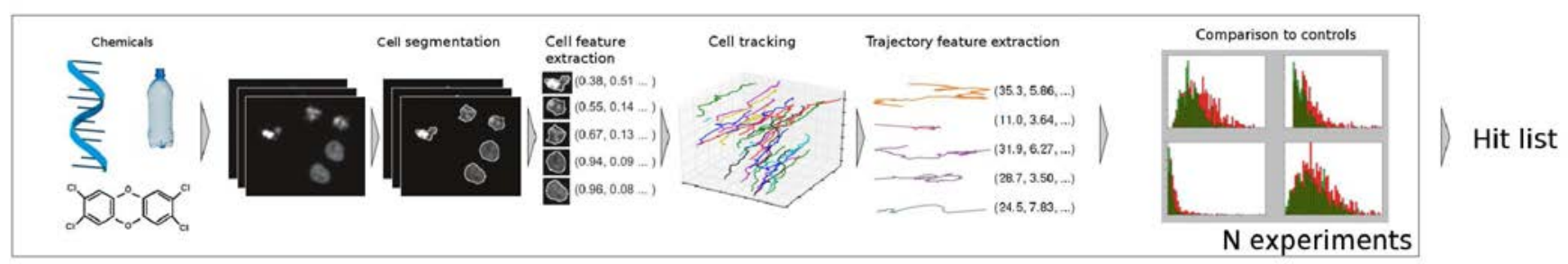

Fig. 1. Overview of MotIW. Images are acquired and segmented. Object features are extracted, which are used for cell tracking. Trajectory feature distributions enables to detect if cell motility was modified by chemical exposure.

are then mapped to a feature space described in section 2.2. Presented in section 2.3, an original statistical procedure then enables the detection of experiments in which single cell motility is significantly different than that in control movies.

\subsection{Cell tracking}

Cell tracking faces several challenges in videos from high content screens like Mitocheck, such as high phenotypic inter-cell variability. Furthermore, the algorithm has to handle apparitions, disparitions, divisions and fusions ${ }^{3}$. Finally, to be applicable in a screening context, as little prior knowledge as possible shall be used regarding cell motion. Therefore, we have extended a non-parametric structured learning approach from [6].

The extended model works as follows : each object in each image is characterized by its position, its orientation and a set of 230 features including geometric, shape and texture features [5]. In this approach, the tracking model matches objects in consecutive frames by assigning them the most likely behaviour in the set of possible matching events

$\mathbf{E}=\{$ move, appear, disappear, split, merge $\}$. The optimal matching is defined as the one that maximizes a likelihood function, which depends on match features and on weights for each feature. This results in a bi-partite graph matching problem; it is solved by an integer linear program. The weights are learned by a support vector machine using annotated trajectories, following the formulation of [6].

The extension compared to [6] lies in the choice of match features. Furthermore, we enabled the tracking model calibration using partial annotation from different experiments. Finally, the original work took neither three object-split, nor three object-merge into account. We added these possibilities to the set of matching events: they are important in a screening context, where aberrant cell divisions may occur.

To validate MotIW's cell tracking procedure, we compare it to Cell Cognition's constrained nearest-neighbour (CNN) tracking algorithm, and to [7] as implemented in Cell Profiler, on a training set with approximately 32,000 matches. The latter come from both control experiments and experiments with significantly altered phenotypes according to [4]. As shown in Table 1, MotIW outperforms the other two methods.

\footnotetext{
${ }^{3}$ This event results from occlusion or segmentation errors.
}

\begin{tabular}{l|c|c} 
Algorithm & Mean recall (\%) & Mean precision(\%) \\
\hline CNN & 72.7 & 62.8 \\
Jaqaman et al. [7] & 78.3 & 73.0 \\
MotIW & 91.1 & 91.5
\end{tabular}

Table 1. Mean recall and precision on all types of matches $\mathbf{E}$ (10-fold cross-validation)

\subsection{Trajectory features}

Once cell trajectories are captured, we extract a set of 15 features from each trajectory. Robust and precise features are needed to account for the partial stochasticity of cell migratory behaviour. We use three types of features, as illustrated in table 2.

On top of classical features such as mean squared displacement, we have designed features to quantify the temporary straightness of a track and the propension of a cell to go from random walk to directed motion. The feature "track curvature" measures temporally local similarity to a line, whereas the feature "diffusion adequation" quantifies how well a diffusive model applies to the track. The feature "movement type" is the power to which time relates to traveled distance [8]. All formulae will be detailed in a longer article about the workflow.

\begin{tabular}{l|l} 
Goal & Examples of feature \\
\hline Particle motion features & $\begin{array}{l}\text { Diffusion coefficient, movement } \\
\text { type }\end{array}$ \\
Other global features & $\begin{array}{l}\text { Track curvature } \\
\text { Mean squared displacement, } \\
\text { Averaged local features signed turning angle }\end{array}$
\end{tabular}

Table 2. Feature types with examples

\subsection{Statistical procedure}

HT screening data is organized in batches of experiments which have been performed simultaneously. Each batch includes a set of negative controls.Due to a non-negligible batch effect, an experiment can only be compared with controls of the same batch in most of the cases.

Let us consider an experiment $i$. Following to trajectory feature extraction, it can be summarized as a set of fea- 

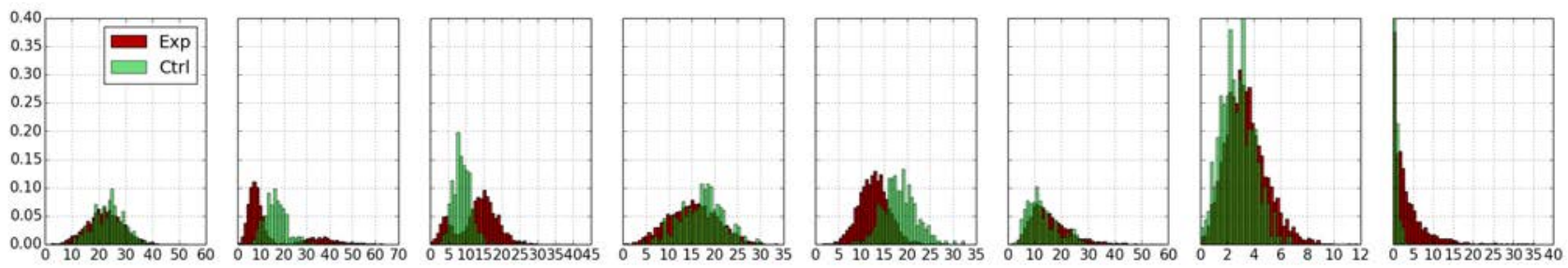

Fig. 2. Comparison of cluster distributions between controls (Ctrl) and experiments (Exp) for the eight trajectory clusters which were identified in the Mitocheck dataset. The clusters are in the same order as in figure 3.

ture distributions. The comparison of these distributions with those of controls from the same batch $B_{i}$, using KolmogorovSmirnov 2-sample test, provides a list of p-values.

A final statistic $S_{i}$ combining the p-values of all features is obtained by Fisher's formula:

$$
S_{i}=-2 \sum_{\text {featuref }} \ln (\mathrm{p}-\mathrm{val}[f])
$$

As the features are not independent, Fisher's combined probability test cannot be directly used. Instead, the same statistic is computed by comparing one control experiment from $B_{i}$ which was not used in the previous comparison, to the other controls from $B_{i}$. Then, the comparison of the distribution of $S$ statistics obtained from control-experiment comparisons, to the distribution of $S$ statistics obtained from controlcontrol comparisons, will permit the computation of empirical p-values. It enables to detect hit experiments with regard to single cell motility. False discoveries are controlled using the Benjamini-Hochberg procedure [9]. An experimental condition, i.e. a siRNA, is detected if more than $50 \%$ condition replicates are detected.

\section{RESULTS}

We first evaluate MotIW on simulated data (see 3.1). We then apply it to the whole genome-wide screen Mitocheck [4], which enables us to identify an ontology of 2D cell trajectories (see section 3.2). In this latter screen, each gene was targeted by three different siRNAs, and each experimental condition was tried in triplicate.

\subsection{Application to a simulated screen}

In a first step, three simple types of cell trajectories were designed: random, fast-random, curbed-directed, and two more complex ones: one in which cells alternate straight-directed motion in $\pm 180^{\circ}$ directions, and one in which cells alternate between random and straight-directed motion.

In a second step, five experimental condition types were designed, each with different trajectory type distributions. "Normal" movies account both for control movies and experiments in which cell motility is similar to that of controls. They contain $80 \%$ of random trajectories, and a mix of the four other trajectory types. This reflects our observation that in real data, all movies contain all possible types of cell trajectories (see fig. 2). All other condition types contain from 50 to $65 \%$ random trajectories, the rest being completed according to the movie type. For example, condition type "fast" is composed of $30 \%$ of fast-random trajectories, $60 \%$ of random trajectories, and a mix of the three other trajectory types. The number of trajectories in each movie was drawn at random in real data, and the number of trajectory of each type in each movie was drawn from the corresponding movie type multinomial distribution.

The third step was the simulation of approximately 50,000 experimental conditions (distributed on 130 plates). Each condition was simulated in three separate experiments, as in a plausible experimental setup. On each plate, between $5 \%$ and $15 \%$ of the experiments were selected to be other than "normal" movies. Our workflow successfully recognized more than $98 \%$ of the experiments, as detailed in table 3 .

\begin{tabular}{l|c|c|} 
& Recall (\%) & Precision (\%) \\
\hline Experiment detection & 99.2 & 98.9 \\
Condition detection & 99.5 & 100.0 \\
\hline \hline Trajectory clustering & $91.4 \pm 2.1$ & $89.4 \pm 4.8$ \\
\hline
\end{tabular}

Table 3. Results from the application of MotIW to simulated data

Our simulation pipeline was also used to estimate how useful our trajectory feature set is to capture the differences between different types of trajectory motion. Hence, we also simulated 500 samples of each of the trajectory types and extracted their features. We then applied k-means to this data set. Due to the fact that many simulation parameters (e.g. each track length) are chosen at random, and that k-means' results are depending on its initialization, we repeated this procedure 10 times. The results are presented in table 3 .

\subsection{Application to the Mitocheck dataset}

When we applied MotIW to the Mitocheck dataset, we were able to identify the experiments which significantly deviate from control movies $(5 \% ; 7,153$ out of 144,909$)$. It amounts to 1,180 genes (out of 17,816 ), some of which are known to 


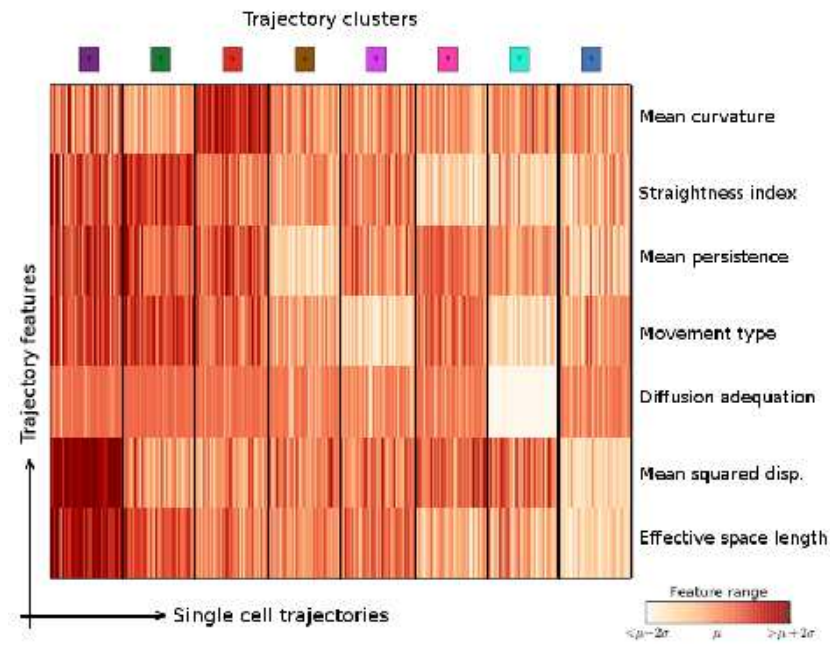

Fig. 3. Characterization of our ontology of trajectories. Each column is a single cell trajectory ; trajectories are grouped by cluster label. 1,000 trajectories were randomly selected per trajectory cluster.

be involved in cellular motility, such as RhoA (Ras homolog family, member A) or CDK5 (cyclin-dependent kinase 5).

Prior to MotIW's design was the question to know whether there exists an ontology of cell trajectories. We could not obtain any valuable clustering from pooling trajectories from the whole dataset, as measured by how the silhouette score and cluster cohesion index vary with respect to the number of clusters [10]. The approach proved successful when pooling trajectories from MotIW's detected experiments, as this smaller dataset is enriched in rare trajectories.

It enabled us to identify eight clusters. Each column of fig. 3 corresponds to one cell trajectory, for which the rows show the standard scores of a subset of features. Interestingly, there is more than speed for differenciating trajectory types. For example, types 2 and 3 present very similar mean squared displacements and space length. However, trajectory curvatures are different: the features mean curvature and straigthness index (another measure of trajectory curvature) are quite distinct between the two clusters.

This can be observed in the Supplementary movie, where cells whose trajectory belongs to cluster 2 (green) are much straighter than those belonging to cluster 3 (red). In this video, cells whose trajectory passed the trajectory quality control have a dot, whose colour corresponds to its cluster as indicated in fig. 3 .

\section{CONCLUSION}

This paper presents a generic methodological framework for studying single cell motility in a high-throughput setup. It combines single cell tracking, newly designed trajectory fea- tures and an original statistical procedure. Furthermore, it is unsupervised: cell motion types were infered from the data without using any prior knowledge. As cell population migration during embryogenesis and metastasis are thought to be led by some leader cell, it is crucial to study single cell motility. Therefore, studying single cell motility ideally complements previous studies on population migration.

In a next step, we are going to apply this workflow to newly generated Environmental Toxicology data in order to identify environmentally relevant chemicals which perturb cell motility.

\section{REFERENCES}

[1] S. Naffar-Abu-Amara, T. Shay, et al., "Identification of novel pro-migratory, cancer-associated genes using quantitative, microscopy-based screening," PLOS ONE, vol. 3, no. 1, pp. e1457, 2008.

[2] K. J. Simpson, L. M. Selfors, et al., "Identification of genes that regulate epithelial cell migration using an siRNA screening approach," Nat. Cell Biol., vol. 10, no. 9, pp. 1027-1038, Sep 2008.

[3] H. Zhang, P. Y. Wu, et al., "An integrative approach for the large-scale identification of human genome kinases regulating cancer metastasis," Nanomedicine, vol. 9, no. 6, pp. 732-736, Aug 2013.

[4] B. Neumann, T. Walter, et al., "Phenotypic profiling of the human genome by time-lapse microscopy reveals cell division genes," Nature, vol. 464, no. 7289, pp. 721-727, Apr 2010.

[5] M. Held, M. H. Schmitz, et al., "CellCognition: timeresolved phenotype annotation in high-throughput live cell imaging," Nat. Methods, vol. 7, no. 9, pp. 747-754, Sep 2010.

[6] X. Lou and F.A. Hamprecht, "Structured Learning for Cell Tracking," in NIPS 2011, 2011.

[7] K. Jaqaman, D. Loerke, et al., "Robust single-particle tracking in live-cell time-lapse sequences," Nat. Methods, vol. 5, pp. 695-702, Aug 2008.

[8] I. F. Sbalzarini and P. Koumoutsakos, "Feature point tracking and trajectory analysis for video imaging in cell biology," J. Struct. Biol., vol. 151, no. 2, pp. 182-195, Aug 2005.

[9] Y. Benjamini and D. Yekutieli, "The control of the false discovery rate in multiple testing under dependency," Annals of Statistics, vol. 29, pp. 1165-1188, 2001.

[10] M. Halkidi, Y. Batistakis, and M. Vazirgiannis, "On clustering validation techniques," Journal of Intelligent Information Systems, vol. 17, pp. 107-145, 2001. 